\title{
COMMENTS
}

In our Special Issue of Volume 14C No. 2, 1980, we published a paper by J. A. Walsh and K. S. Warren entitled 'Selective Primary Health Care: An Interim Strategy for Disease Control in Developing Countries'. Their paper was written in part as one response to the comprehensive Primary Health Care strategy endorsed by all countries which attended the conference held by the WHO at Alma Ata, U.S.S.R., in 1978. In the light of these two events Oscar Gish and Peter Berman have sent us the following two comments on the J. A. Walsh/K. S. Warren paper, which we believe to be sufficiently important to publish. The authors of the original material were invited to respond, and their rejoinders follow these comments together with a note from Mack Lipkin by invitation.

Peter J. M. McEWen

Editor-in-Chief

\section{SELECTIVE PRIMARY HEALTH CARE: OLD WINE IN NEW BOTTLES}

\author{
OSCAR GISH \\ Center for Afroamerican and African Studies, and Center for Research on Economic Developments, \\ The University of Michigan, Lorch Hall, Ann Arbor, MI 48109, U.S.A.
}

\section{BACKGROUND}

Recent events, most notably the first International Conference on Primary Health Care, have highlighted the differences between two classic approaches to many disease control activities. In somewhat oversimplified terms there are, on the one hand, the 'verticalists', favoring categorically specific, hierarchically organized, discrete disease control programs and, on the other, those favoring integrated, 'horizontal' health care delivery systems as the basis of a mixed group of disease control/health promoting activities. In practice, these two extremes are often brought together to a greater or lesser degree.

The verticalists are accused by the integrationists of being overly narrow, of not appreciating the social causation of most disease and hence essentially social nature of its prevention and cure, of seeking only technological ('magic bullet') solutions to problems that are better approached through improved forms of social organization. of attempting to impose external technological hierarchies on peoples rather than working through organized communities, of having failed too often in their past efforts (even their successes are said to have been mostly unique events, e.g., smallpox) and, finally, in their continuing zeal for the vertical campaign approach to be blocking the pathways leading to improved (integrated) health care systems. On the other hand, the integrationists are accused by the verticalists of being idealistic (if not woolly-minded) and unscientific, of trying to impose vague concepts in the social sciences on very real disease vectors, of romanticism and lack of appreciation of hierarchical discipline and, finally, of failing to appreciate the progress that has already been made through specific disease control campaign-type activities.

The International Conference on Primary Health Care held in Alma Ata in September 1978 reflects, within the health sector, an important overall shift in thinking about the nature of Third World underdevelopment. From the end of the Second World War until the 1970s the dominant international view of the solution to the problem of underdevelopment had been what has been termed 'trickle down'. This was taken to mean a process in which a growing national product in a poor country would eventually become large enough so that it would trickle down from the rich to the poor, thus bringing an end to underdevelopment. This trickle down, growth of GNP-based view of development has been replaced over the last decade by the so-called basic needs strategy. This may be defined as the creation of a good standard of nutrition, access to such social services as education and health. full employment (whether based upon paid wages or not) and possibilities for the mass of the population to participate in the social and political processes which affect their lives. The decline of the trickle down, GNP-based view of development has created new challenges and possibilities for the health sector. By extension it requires re-examination of thinking about the bases of health and disease in low income countries (at least) and about the best ways of creating health while attacking disease.

Although few doubt the potential importance of the Alma Ata Conference for the industrialized countries, the more immediate impact is likely to be felt in the less industrialized parts of the world. Of many possible reasons for this, two will be cited here. One is the pressing moral, political, social and economic gap in 
health status that currently exists between the richer and poorer countries. The other is that the limited health resources available to Third World countries make a primary health care approach not only more relevant. but perhaps also essential. The movement away from definition of growth of national product per se as the key component of development means that the improvement of people's health need no longer primarily result from growth; nor need it wait upon that growth, but rather can be accomplished within the framework of existing resource constraints. In fact, it is argued that a healthy, educated, socially involved population is a necessity for true development. The lesson of 'economic growth without development' has been learned by most development theorists (if not by most governments).

The primary health care approach, as developed under the guidance of the World Health Organization, represents the key health services/sector component of the basic needs strategy. The Alma Ata Conference on PHC represented a major international organizational effort to stimulate understanding and adherence on the part of governments to the ideas and practices of primary health care. However, despite the universal rhetorical support being given to the idea of PHC (although different definitions of PHC are being offered), many governments and agencies remain tied to more traditional views of the causes of disease and the best ways of organizing scarce resources within disease control programs. These more traditional views are often expressed in the context of continuing support for categorical disease control programs, and opposition to the integration of such programs into more generalized PHC activities. At least some of the apparent intellectual struggles between verticalists and integrationists seem to be based more on empire protection and building than anything else. Nevertheless, a number of programs originally conceived in more or less vertical terms are exploring new PHC-related strategies for the accomplishment of their goals, e.g. malaria control

It is in this context that the paper by Drs Walsh and Warren, Selective Primary Health Care, An Interim Strategy for Disease Control in Developing Countries', must be considered. It appears to this reviewer that the paper under consideration in fact offers a traditional defense of vertical programs. although the argument is placed into the context of so-called 'selective primary health care'. My comments will follow the structure of the original paper and its sub-headings (except for 'Introductory Material' and 'Concluding Remarks').

\section{INTRODUCTORY MATERIAL}

The lack of analytical rigor which characterizes the paper can be illustrated by close examination of its early paragraphs.

What can be done to help alleviate a nearly unbroken cycle of exposure, disability and death? The best solution. of course, is comprehensive primary health care, defined at the World Health Organization conference held at Alma Ata in 1978 as: the attainment by all peoples of the worid by the year 2000 of a level of health that will permit them to lead a socially and economically productive life
Primary health care includes at least : education concerning prevailing health problems and the methods of preventing and controlling them: promotion of food supply and proper nutrition: an adequate supply of safe water and basic sanitation: maternal and child health care. including family planning: immunization against the major infectious diseases: prevention and control of locally endemic diseases: appropriate treatment of common diseases and injuries: and provision of essential drugs.

The goal set at Alma Ata is above reprodch. yet its very scope makes it unattainable because of the cost and numbers of trained personnel required. Indeed. the World Bank has estimated that it would cost billions of dollars to provide minimal, basic (not comprehensive) healti services by the year 2000 to all the poor in developing countries. The bank's president. Robert McNamara, offered this somber prognosis in his annual report in 1978: Even if the projected-and optimistic-growth rates in the developing world are achieved. some 600 million individuals at the end of the century will remain trapped in absolute poverty. Absolute poverty is a condition of life so characterized by malnutrition, illiteracy, disease, high infant mortality and low life expectancy as to be beneath any reasonable definition of human decency.

Firstly, while the authors themselves (not WHO) introduce the term "comprehensive primary health care', they argue that such comprehensive health services cannot be afforded. Secondly, they slip over from 'health care' to 'health services', which differ especially if they are to be 'comprehensive'. Thirdly, the World Bank is cited as claiming that it would cost billions of dollars to provide initial, basic (not comprehensive) health services by the year 2000 to all the poor in developing countries'. But billions of dollars are already being spent every year in the developing countries on health services. Relatively few developing countries in Asia and Africa of any significant size spend today less than $\$ 5.00$ per capita on modern (Western) health services, and many spend considerably more. Of course the comparable figures for Latin America, the Caribbean, and the Middle East would be considerably higher. It is inconceivable that much less than 15 billion dollars is now being spent annually in the (non-socialist) developing countries for health services, and the actual amount might be double that figure. Fourthly, it is not possible to relate the quotation taken from Mr McNamara to the argument that has been introduced about basic health services (or is it PHC ?). In fact McNamara is arguing just the opposite. He is saying that growth rates alone cannot pull these " 600 million individuals" out of 'absolute poverty'. which is exactly why the basic needs strategy (including PHC) is supported by the World Bank, amongst others.

\section{ESTABLISHING PRIORITIES FOR HEALTH CARE}

\section{The authors write}

Faced with the vast number of health problems of mankind, one immediately becomes aware that all of them cannot be attacked simultaneously. In many regions priorities for instituting control measures must be assigned. and measures that use the limited human and financial resources available most effectively and efficiently must be chosen. Health planning for the developing world thus requires two essential steps: selection of diseases for control and evaluation of different levels of medical intervention from the most comprehensive to the most selective. 
It is the "thus' in the last sentence quoted that is most disturbing; it offers insight into the more traditional types of medical planning. which may be termed 'needs based'. In such exercises, which are never comprehensive, some set of needs or goals are defined (usually in terms of discases, but occasionally overall population coverage); then the resources required to accomplish the chosen goals are set out (usually accepting techniques and 'standards' more or less as practiced in industrialized countries); finally the distance between available and required resources is put forward as a gap that must be bridged so as to meet the plan targets. A common modification of this approach. which is applied by Walsh and Warren, attempts to incorporate 'feasibility of control' into the analysis. In this case "feasibility of control" is taken as the technical possibility of controlling a disease through, say, a vaccine or spraying or chemotherapy.

To the planner working in a real world environment the issue of 'feasibility of control' appears quite differently. The existence of, say, a vaccine by itself means little if the health care network is not in contact with the mass of the population. Of course the planner needs to know, in rough terms anyway, the prevalence of specific diseases and resulting morbidity and mortality in the area for which he or she is responsible, as well as the specific techniques available for prevention and curing specific diseases. However, such information becomes helpful only in the context of the specifics of resource availability and allocation (especially as to their distribution and technical composition). 'Resources' mean not only financial ones or even trained health workers, but also extend to the physical, managerial and administrative infracture as well. Health planners, when not constrained by the politics of the 'big hospital builders', in my view must think in terms of the balanced development of the overall health infrastructure. Whether or not such an infrastructure is in place will drastically alter the overall strategy and feasibility of implementing any particular health care-related activities. The authors are correct 'to reiterate, the most important factor in establishing priorities for endemic infections, even when evaluating diseases with high case rates, is a knowledge of which diseases contribute most to the burden of illness in an area and which are reasonably controllable". However, their definition of "reasonably controllable' is based only upon existing medical and medically related (and/or defined) technologies. To the health planners the concept of reasonably controllable is considerably wider.

In this respect it is important to be aware of the experience of Latin America with the so-called CENDES methodology (named for the Development Studies Center at the Central University of Venezuela). This widely known effort attempted to put into practice a fully formed model for health care planning of the sort put forward in far more simple form by Drs Walsh and Warren. After many years of work and the training of several hundred Latin Americans in the methodology it was concluded in the mid-1970s that planning of this sort was infeasible and thus to be put aside.

The lack of knowledge or possibly selectivity of the authors with regard to past experiences with disease control is well illustrated by the following quotation.
Leprosy and tuberculosis require years of drug therapy and even longer follow-up periods to ensure cure. Instead of attempting immediate, large-scale treatment programs for these infections. the most efficient approach may be to invest in research and development of less costly and more efficacious means of prevention and therapy.

It is probably the case that no disease has been better researched from the perspective of control programs than has been tuberculosis. In fact, the continuing massive problem of tuberculosis in low income countries is testimony to the need for effective national PHC-based health care services, if not necessarily comprehensive PHC in its fullest WHO sense. Many effective, low cost leprosy and tuberculosis programs now operate in developing countries. and much can be learned from them. Drs Walsh and Warren appear to be more knowledgeable about the more fashionable, usually externally supported disease control programs and tuberculosis and leprosy do not fall into that category.

\section{EVALUATING AND SELECTING NEEDED INTERVENTIONS}

The authors argue that, "once diseases are selected for prevention and treatment, the next step is to devise intervention programs of reasonable cost and practicabtlity". There is quite a problem here: either the diseases initially chosen for control were selected in the absence of consideration of "cost and practicability', which would make their initial high priority choice invalid, or some were discarded on cost grounds even in the initial selection process, which makes the overall methodology inconsistent in its application.

The authors then go on to list five different socalled interventions' that are considered to be 'relevant to the world's developing areas'. These are, 'comprehensive primary health care (which includes general development as well as all systems of disease control), basic primary health care, multiple diseasecontrol measures (e.g. insecticides, water supplies), selective primary health care, and research'. Among other things Drs Walsh and Warren seem to imagine a health care world which is in effect a tabula rasa. In practice, of course, every country in the world has a health related system containing a mix of the supposedly discrete areas listed above. It may be objected that these are ideal types only; if so, they cannot be used for the specific review and conclusions that flow from the paper under discussion.

The authors then proceed to discuss and compare these five abstract entities "with emphasis on the relative cost involved in undertaking and maintaining these programs and on the benefits that have accrued'.

[The] analysis relies on reported results from individual studies conducted in various parts of the world. In ad. dition. we have examined estimates of cost and effectiveness in terms of expected deaths averted by each intervention for a model area in Africa. The model area is an agricultural. rural portion of Sub-Saharan tropical Africa with a population of about $500.000(100.000$ are five years old or less). For reference purposes. the average figures for Sub-Saharan Africa will be used: the birth rate is 46 per 
thousand total population. the crude death rate 19 per thousand total population, and the infant mortality rate 147 per thousand live births.

Most of the individual studies referred to are isolated projects, often carried out by external agencies; and as the authors note, their costs and benefits varied immensely. The choice of a 'model area' compounded the problems of analysis in that fairly typical Black African data are used as a point of reference for the entire Third World, presumably including places as diverse as Brasilia, Kingston, Damascus, Colombo and Kuala Lumpur. The studies do not reflect the experiences of countries of the Third World, at least as they function under the real constraints and possibilities of operating health-related activities for entire populations. In any event, the authors confuse diverse pilot project research results, with World Bank or other global estimates, with their own data based upon their African model area and applied to other parts of the world. This fog is difficult to penetrate. It is also worth noting that some sources are referred to which do not support the arguments being made. For example, the 'pilot projects for providing basic healthcare services that have been evaluated vary in their effectiveness in improving the general level of health care. For example, an outside evaluation of primary health service in Ghana revealed... . The authors go on to cite the study's findings of poor services. However, the study examined neither a pilot project nor a 'primary health service'. What it did examine was the ongoing health services in an ordinary rural area of Ghana where, it should be noted, only quite smal resources had been made available to these services.

\section{COMPREHENSIVE VS BASIC PRIMARY HEALTH CARE}

In the view of the authors comprehensive $\mathrm{PHC}$ for everyone "in the near future remains unlikely". They are probably right, at least given their reading of the WHO definition of comprehensive PHC. However, they err in implying that "basic primary health care systems are beyond the reach of low income countries". They cite World Bank estimates on "the cost of furnishing basic health services to all the poor in developing countries by the year 2000 " as being " $\$ 5.4$ to 9.3 billion (in 1978 prices)". These figures include capital and training costs only, but come to only $\$ 5.00$ per capita over a period of 20 years, or $\$ 0.25$ per annum. Of course the greater part of total costs will be the recurrent ones; salaries, drugs, etc. From direct participation this writer knows that it was possible in $1972 / 1973$ to provide a basic PHC network in Tanzania for about $\$ 1.00$ per capita (then) for recurrent costs. This is not to say that one dollar was an optimal figure (there is no absolutely optimal figure and certainly not one that is universally optimal), but in any event the central planning problem is that of spreading scarce resources in an equitable fashion through appropriate technological choices so as to cover whole populations with basic health care services. It is a rather arid exercise to choose some magic number (amount) and expect it to apply equally to 100 Third World countries.

\section{MLLTIPLE DISEASE-CONTROL MEASLRES}

Generally negative conclusions are reached about virtually all activities other than those preferred by the authors: comprehensive PHC "remains unlikely in the near future"; the effectiveness of basic PHC "has not been clearly established"; the financial investment required for sanitation and clean water "is enormous", eradication of various vectors "cannot be considered on the horizon", the costs of nutrition programs are "higher than the cost of medical care alone", and so on. The outlook would be bleak indeed if it wasn't for the possibility of 'selective PHC'.

\section{SELECTIVE PRIMARY HEALTH CARE}

The authors state:

The selective approach to controlling endemic disease in the developing countries is potentially the most cost-effective type of medical intervention. On the basis of high morbidity and mortality and of feasibility of control. a circumscribed number of diseases are selected for prevention in a clearly defined population. Since few programs based on this selective model of prevention and treatment have been attempted, the following approach is proposed. The principal recipients of care would be children up to three years old and women in the childbearing years. The care provided would be measles and diphtheria-pertussistetanus (DPT) vaccination for children over six months old, tetanus toxoid to all women of childbearing age, encouragement of long-term breast feeding, provision of chloroquine for episodes of fever in children under three years old in areas where malaria is prevalent and, finally, oral rehydration packets and instruction.

The authors recommend a mix of health care activities which would rank high on any list of health care priorities in low income countries. What then is different about their proposal? It is that somehow they would provide certain activities to a given population while, again, somehow preventing or denying others. How in practice would this be done? Of course it is possible through rationing, as is the case so commonly now, to simply not make certain vaccines and drugs available to health workers for provision to patients. But even this does not prevent many healthrelated activities from being carried out by resourceful health workers. Perhaps other ways can be found to stop unapproved activities. Of course, politically it would be difficult for most governments to sanction such an approach, especially when the urban and better off population in the country will continue to have access to a wider range of health care activities than is prescribed for the poor. There may be exceptions however, especially when authoritarian States can control their populations as well as those of their health workers who may find it unacceptable to travel to villages and be concerned only with selected problems and persons. It also sometimes is possible for external agencies to operate such programs, at least for specific periods. The authors continue: "These [selective $\mathrm{PHC}$ ] services could be provided by fixed units or by mobile teams visiting once every four to six months in areas where resources were more limited". (It would take us too far afield to question why resources are more limited in some areas of a 
country while being apparently more plentiful in others.) And further:

The cost of fixed units would be similar to that of basic primary health care. although efficiency should be much greater. Cost estimates for a mobile health unit used in the model area in Africa for malaria control and water and sanitation programs were based on an extensive study of the Botswana health services by Gish and Walker. They estimated $\$ 1.26$ as the cost per patient contact in 1974 , on a sample $306-\mathrm{km}$ trip that reached 753 patients; the estimated cost per infant and child death averted was $\$ 200$ to \$250. Medications accounted for 30 to 50 per cent of this cost, but this figure could be decreased with contributions of drugs from abroad or their manufacture within the country.

The authors seem not to understand the cost components of health care programs. They correctly point out that "the cost of fixed units would be similar to that of basic primary health care"; they wrongly assume that "efficiency should be much greater". Presumably they mean that the selective PHC unit would be more productive when confined to those service areas in which it had chosen to function. Apparently then, all health units everywhere would be more efficient if they concerned themselves with only some of their patients ailments and ignored others. In any event, once having invested in the construction and basic staffing of a fixed unit, little is saved by being as rigorously selective as is proposed. It would take too long to spell out all the issues involved in the optimal running of a small rural unit in Africa or Asia, but it is virtually impossible to conceive of circumstances that would justify a fixed unit carrying out only such a relatively narrow range of activities as is proposed by the authors.

The study cited in the last quotation found that the average cost-per-patient-contact of a mobile clinic was almost the same as at a fixed clinic. However the average cost-per-likely-effective-patient-contact (based on the efficaciousness of care including the possibility of patient follow-up) was almost 6 times greater for mobile as compared to fixed clinics. It is useful to note that the cited cost per infant and child death averted is not drawn from the study itself, but apparently calculated from data contained therein and then transferred to the authors" "model area in Africa", possibly in the specific areas of "malaria control and water and sanitation programs".

The authors conclude this section with a quite sensible observation:

Whether the system is fixed or mobile, fiexibility is necessary. The care package can be modified at any time according to the patterns of mortality and morbidity in the area served. Chemotherapy for intestinal helminths, treatment of schistosomiasis and supplementation with new vaccines or treatments as they become available are all types of selective primary health care that could be added or subtracted to this core of basic preventive care. It is important, however, for the service to concentrate on a minimum number of severe problems that affect large numbers of people and for which interventions of established efficacy can be provided at low cost.

Of course this argument can. and should be extended to cover any and all health care interventions in keeping with specific resource and other possibilities in any particular national encironment. This in turn means that the discussion is back to its origins; that is, the need to understand and influence positively the process of health development at a specific time in a specific place. Good.

\section{CONCLUDING REMARKS}

Drs Walsh and Warren seem to ignore the extent to which a health care infrastructure already exists in most countries, even if reaching only a minority of the population of these countries. They also seem not to appreciate the fact that this infrastructure may be expanded quite rapidly during the 1980 s. The basic issue under discussion is the need to find an appropriate match, within the context of specific settings, between the activities properly shown by the authors to be of high priority and the development of broader $\mathrm{PHC}$ activities offering coverage to most of the populations of countries of the Third World.

The paper under discussion does not directly address the nature of the wider development process. The paper also fails to draw adequately upon the historical experiences of Africa, Asia and Latin America with regard to health and health care questions. This lack of both an historical sense and developmental breadth makes impossible the development of a satisfactory approach to the issues under discussion. All of this is compounded by the paper's lack of a social science perspective, and especially as the issues under discussion lie at least as much in this realm as in those of bio-medicine or technology.

Planning for health development and disease control ought not to take the form of either an abstracted 'PHC approach' or another 'vertical campaign'. It is likely that most disease control activities will/should contain elements of both. The problem is to determine the best mix of these elements within the context of specific national historical experiences and possibilities. To the degree there is a primary health care infrastructure capable of supporting appropriate specific disease control activities, to that degree do such programs become feasible and less subject to the cost and other constraints which have defeated so many such programs in the past. This is an area in which much fruitful research remains to be done. The central question to be addressed in such research is determination of the elements dictating the most appropriate levels of 'verticality' or 'integration' to be employed under varying circumstances in the approach to different health and disease problems/ issues. Such determinations would require consideration not only of the specific etiology of individual diseases and the technology available for attacking that disease, but also of the cultural, social, economic, political, administrative and managerial environments in which they exist. In turn, these factors affect the possibilities for positively influencing the problems to be addressed. As a matter of fact, the priority activities advocated by Drs Walsh and Warren are quite compatible with a balanced set of PHC developments: not only are they compatible, but such halanced PHC developments may be essential for the successful extension of these high priority activities to the mass of the population of low income countries. In any event, these matters require informed empirical investigation in the context of particular diseases, pro 
grams and country situations, rather than being issues of subjective belief couched in the concepts of "verticalism' (even when labelled selective primary health care) vs 'integrationism'.
Acknowledgements--Many helpful comments were received from Professors R. N. Grosse and C. M. Wylie of the School of Public Health. The University of Michigan. Of course they do not bear responsibility for the uses to which those comments were put. or anything contained herein.

\title{
SELECTIVE PRIMARY HEALTH CARE: IS EFFICIENT SUFFICIENT "*
}

\author{
Peter A. Berman \\ Department of Agricultural Economics, New York State College of Agriculture and Life Sciences. \\ Cornell University, Ithaca. NY 14853. U.S.A.
}

\begin{abstract}
Developing countries are increasingly using economic evaluation methods to assess and plan their health services. Inappropriate application of these methods may lead to serious errors in developing primary health care strategies. In 'Selective Primary Health Care', Julia Walsh and Kenneth Warren present a logical approach to health planning based on cost-effectiveness techniques. Their paper is a timely example of the risks of using simple technical criteria to plan solutions to complex public health problems. Cost-effectiveness is not a sufficient criterion for planning primary health care. Related issues are discussed in these comments. As an alternative, a multiple-objective approach is suggested.
\end{abstract}

\section{INTRODUCTION}

The major task in planning health services in developing nations is the allocation of limited resources amongst alternative uses. Fundamentally, this is a life and death decision for the populations for whom such decisions are made.

Planners seek to solve the dilemma of such choices by using 'objective' decision criteria. Such criteria do not succeed in removing subjectivity from decisions; they tend to disguise it. The inappropriate application of decision criteria such as cost-effectiveness ratios often leads to simplistic and erroneous solutions to complex problems.

'Selective Primary Health Care' by Julia Walsh and Kenneth Warren [1] is one of a number of papers using cost-effectiveness criteria in primary health care planning that have appeared recently [2-4]. Their 'strategy for disease control in developing countries' is probably infeasible and would clearly be unacceptable as the only government-run rural health program in most developing countries. Yet their conclusions are derived from a logical and, in some ways, useful approach to health planning and the selection of the most cost-effective program alternative.

There are several problems. First, their definition of the planning problem is not relevant to most countries. Second, cost-effectiveness is an insufficient criterion for primary health care program design. Its use by Walsh and Warren reflects an inadequate consideration of the determinants of health program effectiveness and efficiency and of equity objectives in pimary health care. Several lesser errors of assumption and method were also made in their analysis and are discussed below.

Developing countries are increasingly using econ-

*Helpful comments and discussions with Dr Jean-Pierre Habicht and Dr Michael Latham are gratefully acknowledged. omic evaluation methods to assess and plan their health service programs. 'Selective Primary Health Care' offers some valuable guidance. However, planners should take care in applying these techniques beyond their limitations and in drawing conclusions not justified by the available data. This review will hopefully point out some of these constraints.

\section{PRIMARY HEALTH CARE: THE PLANNING PROBLEM}

The broad concept of primary health care (PHC) agreed upon at Alma Ata has been widely reported. Primary health care is described as the means for achieving an ultimate goal, health for all by the year 2000. The Alma Ata conference proposed that PHC should include at least the following activities:

(a) Education concerning prevailing health problems and methods of preventing and controlling them;

(b) Promotion of food supply and proper nutrition;

(c) An adequate supply of safe water and basic anitation;

(d) Maternal and child health care, including family planning;

(e) Immunization against the major infectious diseases:

(f) Prevention and control of locally endemic diseases;

(g) Appropriate treatment of common diseases and injuries;

(h) Provision of essential drugs [5].

The comprehensiveness of this 'minimal' list and the mere two decades set for achieving 'health for all' should be enough to make the most optimistic observer admit secret doubts about the feasibility of this program. While the Alma Ata declaration reflects an important move towards a basic needs strategy, it is also a public recognition of some of the causes of 